\title{
Digital holographic three-dimensional imaging spectrometry
}

\author{
Sirawit Teeranutranont* and Kyu Yoshimori \\ Department of Electrical Engineering and Computer Science, Graduate School of Engineering, \\ Iwate University, Ueda 4-3-5, Morioka, Iwate 020-8551, Japan \\ *Corresponding author: sirawit@ql.cis.iwate-u.ac.jp
}

Received 16 August 2012; revised 12 November 2012; accepted 13 November 2012; posted 14 November 2012 (Doc. ID 174266); published 13 December 2012

\begin{abstract}
A fully interferometric technique for obtaining hypermultispectral components of three-dimensional (3D) images has been tested for a polychromatic object that consists of spatially incoherent, planar light sources of different shapes. Each planar source has different continuous spectra, located at different $3 \mathrm{D}$ positions. The method is based on measurement of a five-dimensional (5D) interferogram. By applying synthetic aperture technique and spectral decomposition to that 5D interferogram, one obtains a set of complex holograms of different spectral components. From these holograms, 3D images of multiple spectral components have been retrieved. A decomposed continuous spectrum of each planar light source is also shown to demonstrate the potential applicability to identify materials of a particular part of an object under illumination by white light. (C) 2012 Optical Society of America
\end{abstract}

OCIS codes: $\quad 090.1995,300.6300,110.3175$.

\section{Introduction}

Attempts to develop a passive interferometric techniques to obtain monochromatic three-dimensional (3D) images [1-4], and two-dimensional (2D) images of different spectra [5-7] have been reported in the past decades. Reports on interferometric techniques to obtain 3D images of many spectral components are rare, however [8-13]. We have considered a method to obtain simultaneously 3D spatial information and spectral information for a usual polychromatic object by use of a fully passive interferometric technique.

For this purpose, we propose a method that uses a suitably designed interferometer and signal processing, including synthetic aperture technique and spectral decomposition to recover a set of complex holograms. The method uses only interferometric techniques and signal processing applied to the interferogram generated by propagated light from the measured object. We have reported experimental results in retrieving the $3 \mathrm{D}$ position and spectrum of a

$1559-128 \mathrm{X} / 13 / 01 \mathrm{~A} 388-09 \$ 15.00 / 0$

(C) 2013 Optical Society of America monochromatic point source [14], and a pair of monochromatic point sources of different frequencies, located at different positions [15]. This paper presents experimental results of the method applied to retrieve the spectral components of $3 \mathrm{D}$ images of the spatially incoherent, polychromatic light source distribution, each point of which could have different continuous spectrum. We call the method the digital holographic 3D imaging spectrometry.

Section 2 summarizes the procedure of our method in connection with the experimental system. Section $\underline{3}$ shows our detailed experimental setup and object to be measured. The results of the experiment are shown in Section 4. New results include a set of 3D images, continuous spectral profiles of the polychromatic light source distribution, and decomposed continuous spectra at particular 3D positions of polychromatic object. The work concludes with Section 5 .

\section{Principle of Method}

This section presents the principle of the digital holographic 3D imaging spectrometry. We begin by introducing the concept of the measurement system, a main part of which is called the two-wavefront 
folding interferometer. This interferometer measures five-dimensional (5D) interferogram, which contains $5 \mathrm{D}$ spatial coherence function. We then show the procedure of synthetic aperture technique to generate reduced-volume (3D) interferogram, spectral decomposition to obtain a set of crossspectral densities for different spectral bands. Finally, from these incoherent complex holograms, the 3D spatial information of the light source distribution at each spectral component may be obtained.

\section{A. Measurement of 5D Interferogram (5D Spatial Coherence Function)}

Figure 1 illustrates the schematic of the twowavefront folding interferometer that is used in our experimental system. The polychromatic light source distribution under measurement $(\mathrm{S})$ is placed on the $x-y$ stage. The optical field propagating from the light source distribution is divided into two parts by the beam splitter (BS). Each wavefront, split by the $\mathrm{BS}$, is reversed from up to down or right to left by the prism $\mathrm{P}$ or $\mathrm{P}^{\prime}$. Those light waves are superposed on BS again. Then an interference-fringe pattern is created. This interference pattern is recorded by the CCD camera at the observation plane. By moving the piezoelectric transducer (PZT) on which the prism $\mathrm{P}$ is placed, an optical path difference along the optical axis ( $z$-direction) is introduced. By moving the $x-y$ stage and the $z$ stage (PZT) stepwise, the relative configuration of the two divided wavefronts are changed and, then, the interference pattern at each configuration is recorded. In this way, the $5 \mathrm{D}$ spatial coherence function is measured.

To analyze the spatial coherence function recorded in the interferogram, we write $\boldsymbol{r}_{s}=\left(x_{s}, y_{s}, z_{s}\right)$ as a particular point on the 3D polychromatic light source distribution. We denote the angular frequency $\omega_{s}=c k_{s}$, with $c$ standing for the speed of light in free space, and $k_{s}=2 \pi / \lambda_{s}$ the wave number of concern, with $\lambda_{s}$ denoting the wavelength.

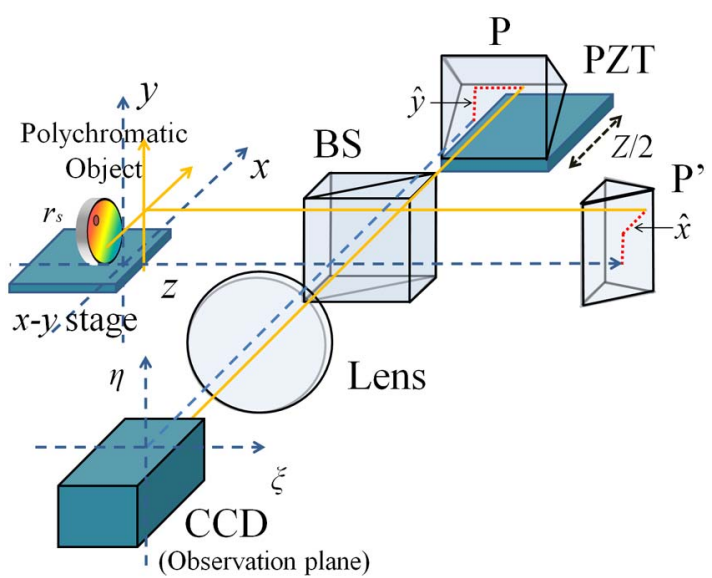

Fig. 1. (Color online) Schematic of the two-wavefront folding interferometer. The optical axes of the interferometer are indicated by solid lines that intersect at the center of the apexes of the rightangle prisms. The Cartesian coordinate system, fixed on the object, is shown by broken lines.
Let $\hat{x}$ and $\hat{y}$ be the positions of the apexes of the prism $\mathrm{P}^{\prime}$ and $\mathrm{P}$, respectively, and let $z_{0}$ be the optical length from the observation plane to the origin of the Cartesian coordinate system. The position of this observation plane is adjusted by the lens $\mathrm{L}$ onto apex of the prism $\mathrm{P}^{\prime}$. Here, $Z$ denotes the optical path difference introduced by PZT. The measured interference pattern, called elementally interference pattern, denoted $I(x, y, \hat{x}, \hat{y}, Z)$ over the observation plane $z=z_{0}$, is given by

$$
\begin{aligned}
i(x, y, \hat{x}, \hat{y}, z)= & \frac{1}{4}\left\{\Gamma(\boldsymbol{r}, \boldsymbol{r})+\Gamma\left(\boldsymbol{r}^{\prime}, \boldsymbol{r}^{\prime}\right)+\Gamma\left(\boldsymbol{r}^{\prime}, \boldsymbol{r}\right)+\Gamma\right. \\
& \left.*\left(\boldsymbol{r}^{\prime}, \boldsymbol{r}\right)\right\},
\end{aligned}
$$

where the asterisk denotes the complex conjugate. Here, we suppress the time $t$ in $\Gamma$ since the optical field is stationary. In Eq. (1),$\Gamma\left(\boldsymbol{r}^{\prime}, \boldsymbol{r}\right)=$ $\left\langle V^{*}\left(\boldsymbol{r}^{\prime}, t\right) V(\boldsymbol{r}, t)\right\rangle$ stands for the spatial coherence function of the optical field $V(r, t)$ at a pair of points $r$ and $\boldsymbol{r}^{\prime}$. The 3D position vectors $\boldsymbol{r}=\left(x, y-2 \hat{y}, z_{0}+Z\right)$ and $r^{\prime}=\left(x-2 \hat{x}, y, z_{0}\right)$ are superposed points of the optical field, which are reversed in a different way by the prism $\mathrm{P}$ and $\mathrm{P}^{\prime}$, respectively, returned to $\mathrm{BS}$ from the two interferometer arms. For spatially incoherent polychromatic source distribution, the spatial coherent function $\Gamma\left(\boldsymbol{r}^{\prime}, \boldsymbol{r}\right)$ [1], which appears in the interference pattern in Eq. (1), is expressed as

$$
\Gamma\left(\boldsymbol{r}^{\prime}, \boldsymbol{r}\right)=\int S_{\omega}\left(\boldsymbol{r}_{s}\right) \exp \left(i k\left(r-r^{\prime}\right)\right) d^{3} r_{s} \mathrm{~d} \omega,
$$

where $r=\left|\boldsymbol{r}-\boldsymbol{r}_{s}\right|$ and $r^{\prime}=\left|\boldsymbol{r}^{\prime}-\boldsymbol{r}_{s}\right| . S_{\omega}\left(\boldsymbol{r}_{s}\right)$ denotes the spectral intensity of the light source at $r_{s}$. We now use paraxial approximation and assume that $z_{0} \gg Z$, where $z=z_{0}-z_{s}$ is the optical depth of the light source. Then $r$ and $r^{\prime}$, appeared on the right-hand side of Eq. (2), are expressed by

$$
\begin{aligned}
& r \cong z+Z+\frac{\left(x-x_{s}\right)^{2}+\left(y-2 \hat{y}-y_{s}^{2}\right)}{2 z}, \\
& r \cong z+\frac{\left(x-2 \hat{x}-x_{s}\right)^{2}+\left(y-y_{s}^{2}\right)}{2 z},
\end{aligned}
$$

which yields

$$
r-r^{\prime} \cong Z+\frac{2 \hat{x}\left(x-x_{s}\right)-2 \hat{y}\left(y-y_{s}\right)}{z}+\frac{-(2 \hat{x})^{2}+(2 \hat{y})^{2}}{2 z} .
$$

Substitution of Eq. (ㅁ) into Eq. (2 $)$ gives

$$
\begin{aligned}
& \Gamma\left(x-2 \hat{x}, y, z_{0} ; x, y-2 \hat{y}, z_{0}+Z\right) \\
& =\int S_{\omega}\left(r_{s}\right) \exp (i k Z) \exp \left[\frac{2 \hat{x}\left(x-x_{s}\right)-2 \hat{y}\left(y-y_{s}\right)}{z}\right] \\
& \quad \times \exp \left[\frac{-(2 \hat{x})^{2}+(2 \hat{y})^{2}}{2 z}\right] d^{3} r_{s} d \omega .
\end{aligned}
$$

Because the motion of the $x-y$ stage is $2 \mathrm{D}$ within the $x-y$ plane, and the origin of the Cartesian 
coordinate system is fixed on the $x-y$ stage, the origin of the $x-y$ plane does not always coincide with the origin of the coordinate system $(\xi, \eta)$ defined over the observation plane. The relationship between $(x, y)$ coordinates and $(\xi, \eta)$ coordinates is written as

$$
\begin{aligned}
& \xi=x-\hat{x}, \\
& \eta=y-\hat{y} .
\end{aligned}
$$

By substituting Eq. (6a) and Eq. (6b) into Eq. (5), the spatial coherence function can be rewritten in terms of the five independent variables $(\xi, \eta, \hat{x}, \hat{y}$, and $Z$ ) as follows:

$$
\begin{aligned}
& \Gamma\left(-\hat{x}+\xi, \hat{y}+\eta, z_{0} ; \hat{x}+\xi,-\hat{y}+\eta, z_{0}+Z\right) \\
& =\int S_{\omega}\left(\boldsymbol{r}_{s}\right) \exp (i k Z) \\
& \quad \times \exp \left[i k \frac{2 \hat{x}\left(\xi-x_{s}\right)-2 \hat{y}\left(\eta-y_{s}\right)}{z}\right] d^{3} r_{s} d \omega .
\end{aligned}
$$

The whole data set $I(x, y, \hat{x}, \hat{y}, Z)$, that is, a set of elementally interference pattern in Eq. (1), should be regarded as a 5D interferogram. This may be illustrated as arranging the 2D elemental interference patterns, in the $3 \mathrm{D}(\hat{x}, \hat{y}, Z)$ space as shown in Fig. 2.

\section{B. Synthetic Aperture Technique to Generate} Reduced-Volume (3D) Interferogram

To compute the reduced-volume (3D) interferogram, we apply the synthetic aperture technique to the 5D interferogram obtained by the two-wavefront folding interferometer as described earlier. Figure $\underline{3}$ shows the example of reduced-volume (3D) interferogram. We will find that this interferogram is suitable for retrieving the source information under observation.

The first step in generating a volume interferogram is to choose one pixel value from each elementally interference pattern according to the following selection rule:

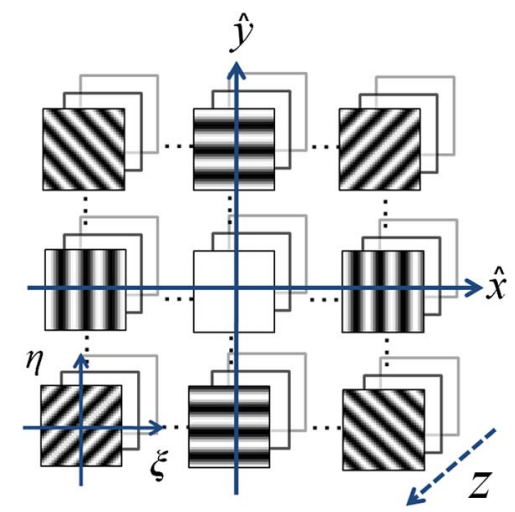

Fig. 2. (Color online) Example of an entire interference data set as the 5D interferogram: position on each 2D elemental interference image $(\xi, \eta), x, y$ position of $\mathrm{P}^{\prime}, \mathrm{P}$ apex $(\hat{x}, \hat{y})$, and optical path difference along the $z$-direction $(Z)$.

$$
\begin{aligned}
& \xi=\hat{x}, \\
& \eta=-\hat{y} .
\end{aligned}
$$

The selected data contains the spatial coherence function. We then introduce the new parameters

$$
\begin{aligned}
& X=2 \hat{x}, \\
& Y=2 \hat{y},
\end{aligned}
$$

to rearrange the selected data. The rearranged data set forms the reduced-volume interferogram and contains a 3D spatial coherence function of the form:

$$
\begin{aligned}
\Gamma\left(0,0, z_{0} ; X, Y, z_{0}+Z\right)= & \int S_{\omega}\left(\boldsymbol{r}_{s}\right) \exp (i k Z) \\
& \times \exp \left(i k_{x} X+i k_{y} Y\right) \\
& \times \exp \left[i k \frac{X^{2}+Y^{2}}{2 z}\right] d^{3} r_{s} d \omega
\end{aligned}
$$

where $k_{x}=-k x_{s} / z$ and $k_{y}=-k y_{s} / z$ are the $x$ and $y$ components of the wave-number vector $k$ of the plane wave propagating from the direction of a particular point $\boldsymbol{r}_{s}$ on the source to the center of the observation plane.

We next compute a reduced-volume (3D) interferogram from the originally measured 5D interferogram. We find that spectral information at different wavelengths may be separated from the reducedvolume interferogram. Therefore, we will show the spectral decomposition technique to obtain a set of cross-spectral densities for every spectral component in Subsection 2.C.

\section{Recovery of a Set of Complex Holograms for Many Spectral Bands}

For the stationary optical field, the mutual correlation function, denoted $\Gamma\left(\boldsymbol{r}^{\prime}, \boldsymbol{r} ; \tau\right)$, at two space-time points $(\boldsymbol{r}, t+\tau)$ and $\left(\boldsymbol{r}^{\prime}, t\right)$ is generally expressed in terms of the cross-spectral density $W_{\omega}\left(\boldsymbol{r}^{\prime}, \boldsymbol{r}\right)$ as

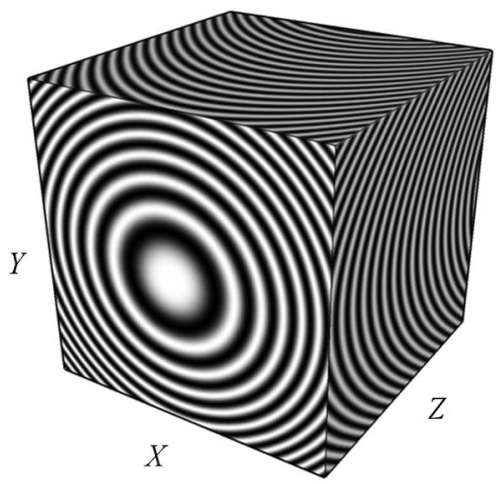

Fig. 3. Example of the reduced-volume interferogram for monochromatic point source located at the origin of the Cartesian coordinate system. 


$$
\begin{aligned}
\Gamma\left(\boldsymbol{r}^{\prime}, \boldsymbol{r} ; \tau\right) & =\left\langle V^{*}\left(\boldsymbol{r}^{\prime}, t\right) V(\boldsymbol{r}, t+\tau)\right\rangle \\
& =\int_{0}^{\infty} W_{\omega}\left(\boldsymbol{r}^{\prime}, \boldsymbol{r}\right) \exp (-i \omega \tau) d \omega,
\end{aligned}
$$

where the angular bracket denotes the ensemble averages, the asterisk denotes the complex conjugate, and $\tau$ is the time difference. Setting $\tau=0$ yields

$$
\Gamma\left(\boldsymbol{r}^{\prime}, \boldsymbol{r}\right)=\int_{0}^{\infty} W_{\omega}\left(\boldsymbol{r}^{\prime}, \boldsymbol{r}\right) d \omega
$$

Thus, for the stationary field, the spatial coherence function is given as an integral of spectral components (i.e., cross-spectral densities). By comparing Eq. (12) with Eq. (10), we find that Eq. (10) may be rewritten in the following form:

$$
\Gamma\left(\boldsymbol{R}_{0}, \boldsymbol{R}_{0}+\boldsymbol{\rho}\right)=\int_{0}^{\infty} W_{\omega}\left(\boldsymbol{R}_{0}, \boldsymbol{R}_{0}+\boldsymbol{\rho}\right) d \omega
$$

with the cross-spectral density defined as

$$
\begin{aligned}
W_{\omega}\left(\boldsymbol{R}_{0}, \boldsymbol{R}_{0}+\boldsymbol{\rho}\right)= & \int S_{\omega}\left(\boldsymbol{r}_{s}\right) \exp (i k Z) \exp \left(i k_{x} X+i k_{y} Y\right) \\
& \times \exp \left[i k \frac{X^{2}+Y^{2}}{2 z}\right] d^{3} r_{s}
\end{aligned}
$$

In Eq. (14), $\boldsymbol{R}_{\mathbf{0}}=\left(0,0, z_{0}\right)$ denotes the center of the observation plane and $\rho=(X, Y, Z)=\left(\rho_{\perp}, Z\right)$. We now define the point $\boldsymbol{r}^{\prime \prime}=\left(\boldsymbol{R}_{0}+\boldsymbol{\rho}_{\perp}\right)$ on the observation plane $z=z_{0}$. This point $r^{\prime \prime}$ is actually the position of $r$ shifting to $Z=0$. Within the paraxial approximation, the cross-spectral density $W_{\omega}\left(\boldsymbol{R}_{0}, \boldsymbol{R}_{0}+\boldsymbol{\rho}_{\perp}\right)$ on the observation plane is related to $W_{\omega}\left(\boldsymbol{R}_{0}, \boldsymbol{R}_{0}+\boldsymbol{\rho}\right)$ as

$$
W_{\omega}\left(\boldsymbol{R}_{0}, \boldsymbol{R}_{0}+\boldsymbol{\rho}\right)=\exp (i k Z) W_{\omega}\left(\boldsymbol{R}_{0}, \boldsymbol{R}_{0}+\boldsymbol{\rho}_{\perp}\right) .
$$

Here, we obtain the relation between the measured spatial coherence function and the crossspectral density over the observation plane by

$$
\Gamma\left(\boldsymbol{R}_{0}, \boldsymbol{R}_{0}+\boldsymbol{\rho}\right)=\int_{0}^{\infty} W_{\omega}\left(\boldsymbol{R}_{0}, \boldsymbol{R}_{0}+\boldsymbol{\rho}_{\perp}\right) \exp (i k Z) d \omega .
$$

It is then clear that Eq. (16) may be inverted to express the cross-spectral density as the Fourier transform of the reduced 3D spatial correlation function:

$$
W_{\omega}\left(\boldsymbol{R}_{0}, \boldsymbol{R}_{0}+\boldsymbol{\rho}_{\perp}\right)=\frac{1}{2 \pi} \int \Gamma\left(\boldsymbol{R}_{0}, \boldsymbol{R}_{0}+\boldsymbol{\rho}\right) \exp (-i k Z) d Z .
$$

Actually, the integrand is taken over the actual extension of the interferogram with respect to $Z$. From Eq. (17), we write the cross-spectral density at the observation plane in terms of the spectral intensity of the light source $S_{\omega}\left(\boldsymbol{r}_{s}\right)$ at $\boldsymbol{r}_{s}$ :

$$
\begin{aligned}
W_{\omega}^{\left(z_{0}\right)}\left(\boldsymbol{\rho}_{\perp}\right)= & W_{\omega}\left(\boldsymbol{R}_{0}, \boldsymbol{R}_{0}+\boldsymbol{\rho}_{\perp}\right) \\
= & \int S_{\omega}\left(\boldsymbol{r}_{s}\right) \exp \left(i k_{x} X+i k_{y} Y\right) \\
& \times \exp \left[i k \frac{X^{2}+Y^{2}}{2 z}\right] d^{3} r_{s} .
\end{aligned}
$$

Here, Eq. (18) allows us to compute the crossspectral density across the observation plane. This cross-spectral density is equivalent to a complex hologram of the spectrum of concern. Then, we can retrieve $3 \mathrm{D}$ images in those spectra by making use of the propagation law of the angular cross-spectral density as described in Subsection 2.D.

D. Retrieval of 3D Image for Many Spectral Bands

To retrieve the spectral components of the $3 \mathrm{D}$ images, we are now in a position to formulate the principle for retrieving the cross-spectral density across source planes on the basis of the propagation law of the 2D Fourier transform of cross-spectral density.

From Eq. (18), the cross-spectral density across the observation plane can be represented by a crosscorrelation of monochromatic optical field at two points $\boldsymbol{r}^{\prime \prime}$ and $\boldsymbol{r}^{\prime}$ :

$$
\begin{aligned}
W_{\omega}^{\left(z_{0}\right)}\left(\boldsymbol{\rho}_{\perp}\right) & =W_{\omega}\left(0,0, z_{0} ; \boldsymbol{\rho}_{\perp}, z_{0}\right) \\
& =\left\langle U_{\omega}^{*}\left(0,0, z_{0}\right) U_{\omega}\left(\boldsymbol{\rho}_{\perp}, z_{0}\right)\right\rangle .
\end{aligned}
$$

where $U_{\omega}\left(\rho_{\perp}, z_{0}\right)$ denotes the monochromatic component of the complex amplitude of optical field over a reference plane at $z=z_{0}$. The $2 \mathrm{D}$ Fourier transforms of Eq. (19) gives a definition of the angular crossspectral density defined in the $\boldsymbol{k}_{\perp}$ space:

$$
\begin{aligned}
\tilde{W}_{\omega}^{\left(z_{0}\right)}\left(\boldsymbol{k}_{\perp}\right) & =\frac{1}{2 \pi} \int W_{\omega}^{\left(z_{0}\right)}\left(\boldsymbol{\rho}_{\perp}\right) \exp \left(-i \boldsymbol{k}_{\perp} \cdot \boldsymbol{\rho}_{\perp}\right) d^{2} \rho_{\perp} \\
& =\left\langle U_{\omega}^{*}\left(0,0, z_{0}\right) \tilde{U}_{\omega}\left(\boldsymbol{k}_{\perp}, z_{0}\right)\right\rangle,
\end{aligned}
$$

where we write $\boldsymbol{k}_{\perp}=\left(k_{x}, k_{y}\right)$ and $\boldsymbol{k}=\left(k_{x}, k_{y}, k_{z}\right)=$ $\left(\boldsymbol{k}_{\perp}, k_{z}\right)$. From Eq. (20), the cross-spectral density defined in the Fourier space, across a certain source plane $\left(z=z^{\prime}<z_{0}\right)$ can be retrieved by the back propagation of the angular cross-spectral density toward the source. The relationship between the angular cross-spectral densities at $z=z_{0}$ and $z=z_{0}-z^{\prime}$ are expressed as

$$
\tilde{W}_{\omega}^{\left(z_{0}-z\right)}\left(\boldsymbol{k}_{\perp}\right)=\exp \left(-i k_{z} z\right) \tilde{W}_{\omega}^{\left(z_{0}\right)}\left(\boldsymbol{k}_{\perp}\right),
$$

where $k_{z}=\left[k^{2}-k_{\perp}^{2}\right]^{1 / 2}$ is the component of the wave number vector $\boldsymbol{k}$ as a function of $\boldsymbol{k}_{\perp}$. We neglect the evanescent wave because we restrict $k_{\perp}<k$. The cross-spectral density across the source plane is 


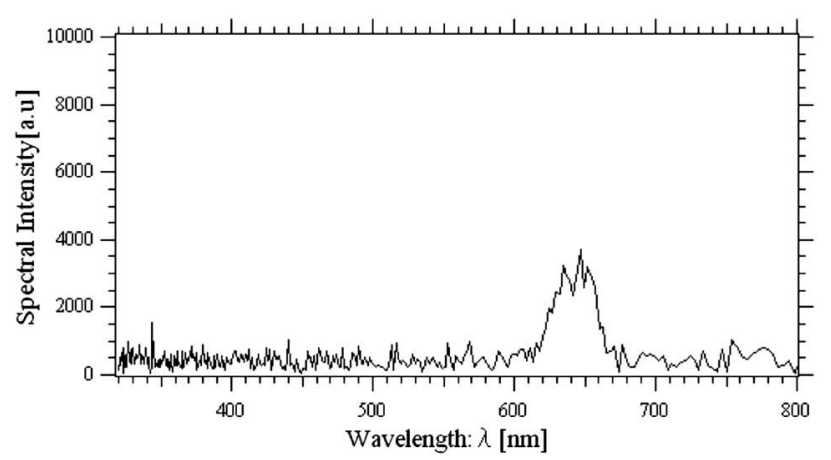

(a)

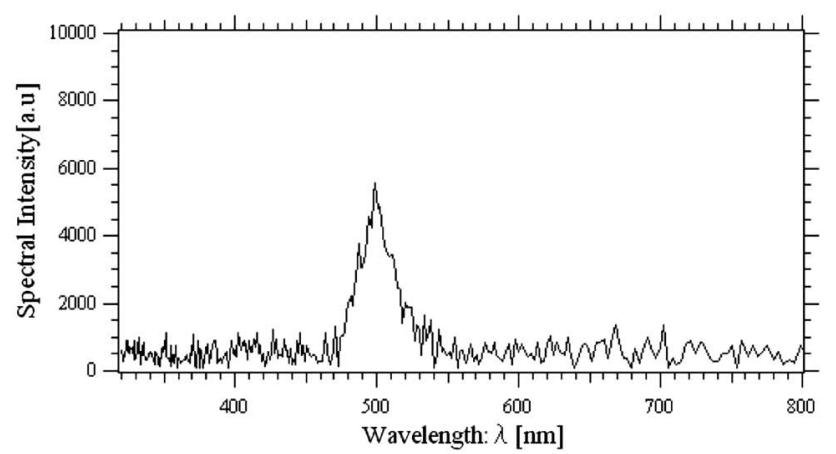

(b)

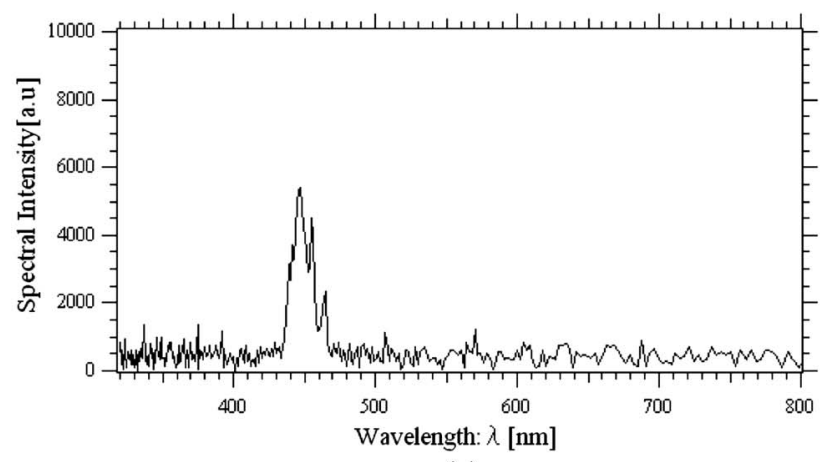

(c)

Fig. 4. Continuous spectral profile of each light source: (a) S1, (b) S2, and (c) S3.

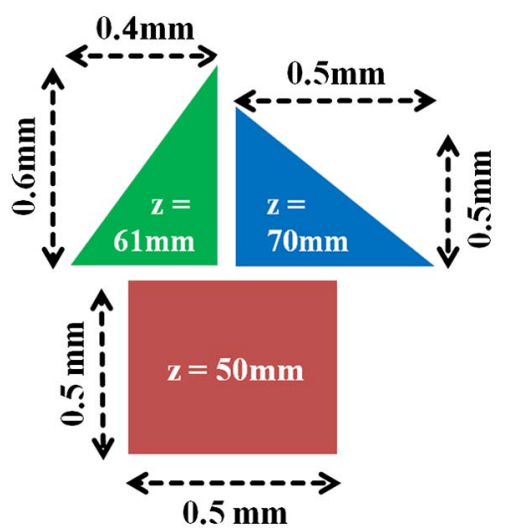

Fig. 5. (Color online) Shape of the measured light sources: S1 (down), S2 (left-up), and S3 (right-up).
Table 1. Specification of Planar Light Sources

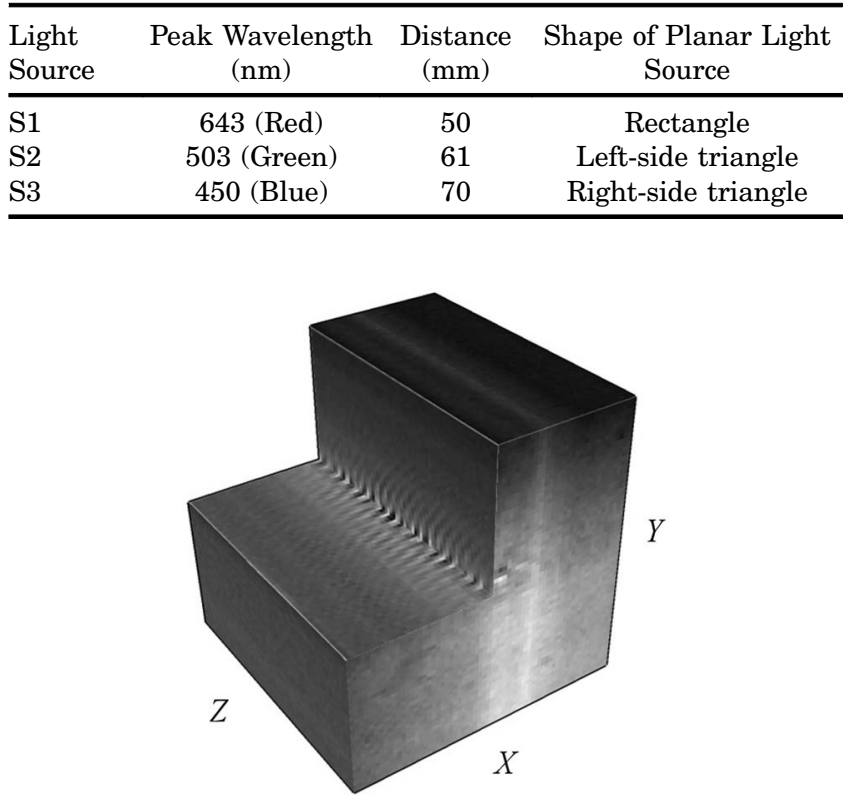

Fig. 6. Reduced-volume (3D) interferogram for the spatially incoherent light source distribution. The center of the volume interferogram shows the inner interference fringe.

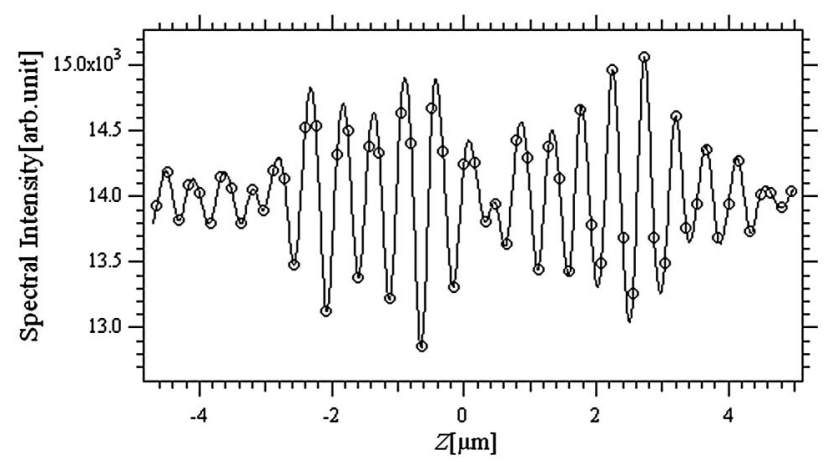

Fig. 7. One-dimensional interferogram of intensity profile plotted with respect to $Z$ along center line of the volume interferogram.

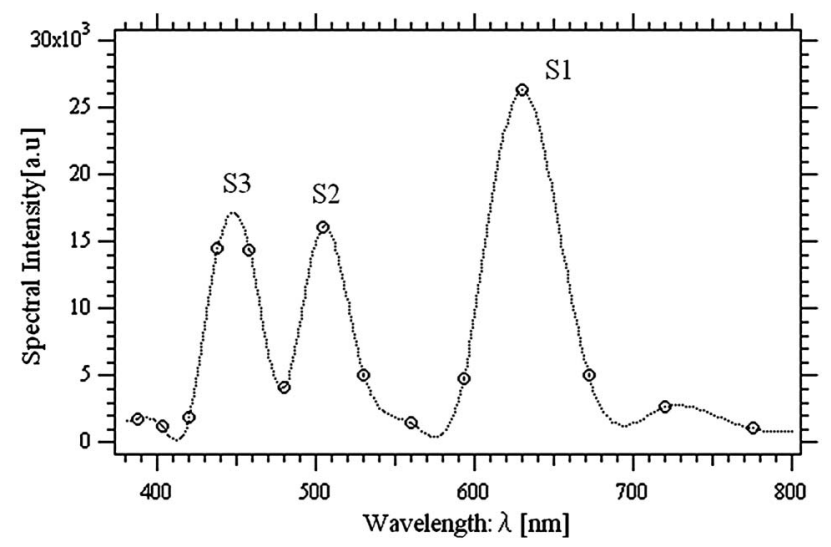

Fig. 8. Continuous spectral profile recorded on the observation plane. This is obtained by taking Fourier transform of the reduced-volume interferogram with respect to $Z$. 
retrieved with Eq. (21) and the 2D inverse Fourier transform,

$$
\begin{aligned}
W_{\omega}^{\left(z_{0}-z\right)}\left(\boldsymbol{\rho}_{\perp}\right)= & \frac{1}{2 \pi} \int \tilde{W}_{\omega}^{\left(z_{0}\right)}\left(\boldsymbol{k}_{\perp}\right) \exp \left(-i\left(\boldsymbol{k}_{\perp} \cdot \boldsymbol{\rho}_{\perp}\right.\right. \\
& \left.\left.-k_{z} z\right)\right) d^{2} \boldsymbol{\rho}_{\perp} \\
= & \left\langle U_{\omega}^{*}\left(0,0, z_{0}\right) U_{\omega}\left(\boldsymbol{\rho}_{\perp}, z_{0}-z\right)\right\rangle .
\end{aligned}
$$

Thus,

$$
\begin{aligned}
\left|W_{\omega}^{\left(z_{0}-z\right)}\left(\rho_{\perp}\right)\right| & =\left|\left\langle U_{\omega}^{*}\left(0,0, z_{0}\right) U_{\omega}\left(\rho_{\perp}, z_{0}-z\right)\right\rangle\right| \\
& \propto\left|U_{\omega}\left(\rho_{\perp}, z_{0}-z\right)\right| .
\end{aligned}
$$

Because this cross-spectral density is proportional to the spectral component of the complex amplitude
$\left|U_{\omega}\left(\rho_{\perp}, z_{0}-z\right)\right|$ across the source plane, it is possible to retrieve the spectral component of a 3D image of the light source by a conventional angular spectrum technique.

In this manner, the cross-spectral density and the spatial distribution of the spectral density, namely, the 3D image at each spectral component, can be obtained.

\section{Experiment}

In this experiment, we used three different color LEDs as the measured objects. Figure 4 shows a continuous spectral profile of each LED, which is measured by Fourier transform spectrometry, in which the spectral resolution is $61.0948 \mathrm{~cm}^{-1}$ and the spectral range is $3.125 \times 10^{4} \mathrm{~cm}^{-1}$. To create three

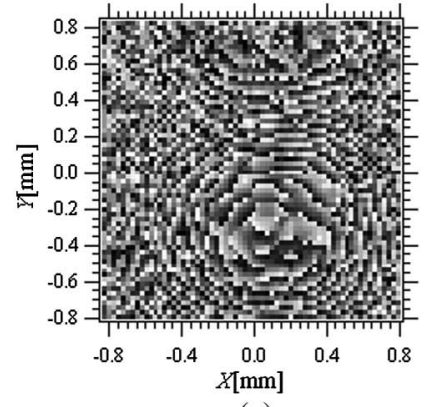

(a)

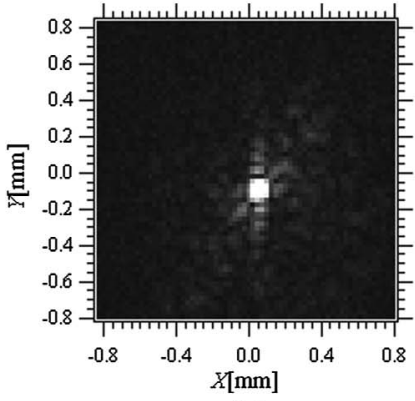

(b)

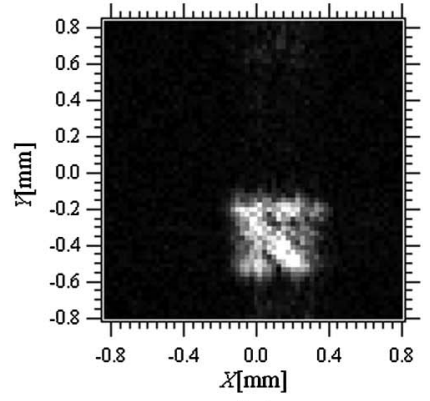

(c)

Fig. 9. (a) Phase distribution and (b) absolute value of the cross-spectral density at spectral peak $\lambda=630 \mathrm{~nm}$. (c) In-focus images over $x-y$ plane at $z=50 \mathrm{~mm}$.

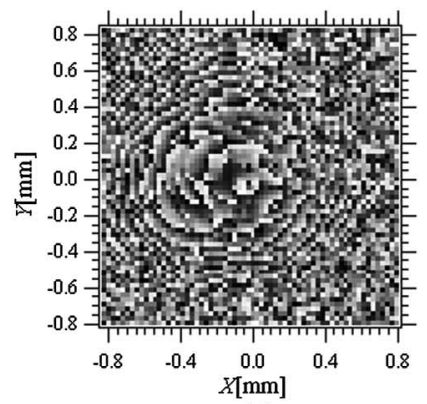

(a)

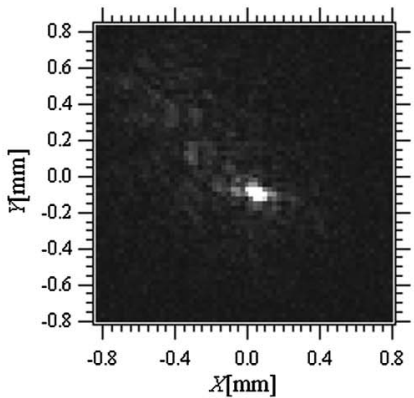

(b)

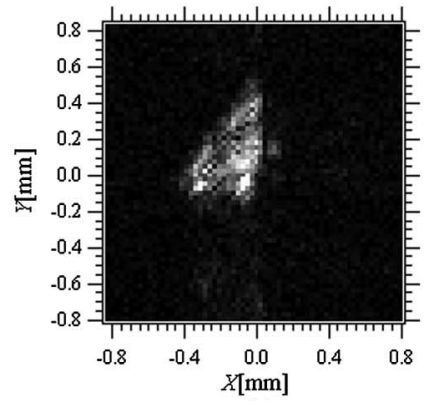

(c)

Fig. 10. (a) Phase distribution and (b) absolute value of the cross-spectral density at spectral peak $\lambda=504 \mathrm{~nm}$. (c) In-focus images over $x-y$ plane at $z=61 \mathrm{~mm}$.

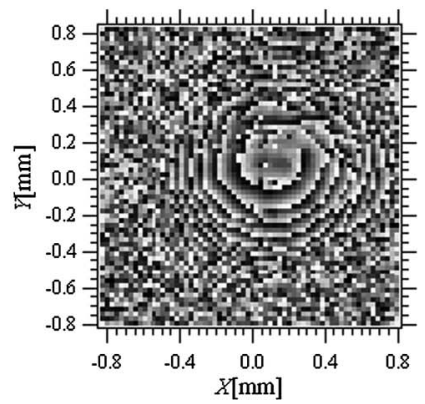

(a)

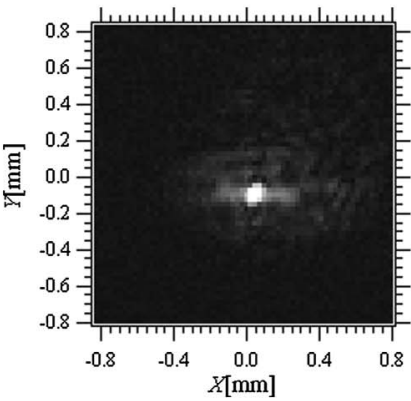

(b)

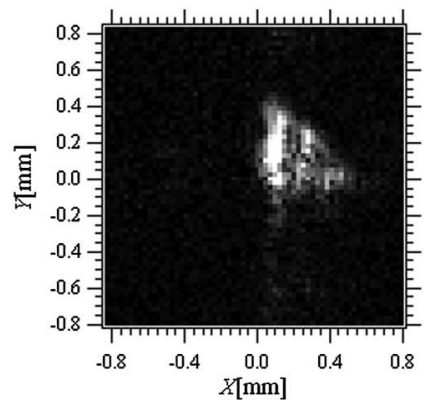

(c)

Fig. 11. (a) Phase distribution and (b) absolute value of the cross-spectral density at spectral peak $\lambda=448 \mathrm{~nm}$. (c) In-focus images over $x-y$ plane at $z=70 \mathrm{~mm}$. 
planar light sources of different shapes, we attached acrylic sticks to the three surfaces of light sources. The cross sections on the other side of the three sticks are fabricated to different shapes; a rectangle and two different triangles, as shown in Fig. 5 . The planar light sources are set up on the $x-y$ stage of the two-wavefront folding interferometer. The $x-y$ stage is moved along the $x$ and $y$ axes 64 times in each direction for $12.9 \mu \mathrm{m}$ in every step. Additionally, the prism $\mathrm{P}$, placed on the PZT, is also moved 64 times in $z$-direction for $0.08 \mu \mathrm{m}$ in every step. There are 262,144 total steps in our experiment.

The movement interval and number of steps of the $x-y$ stage are equal to the pixel size and pixel number of the CCD camera, respectively. These parameters determine the transverse resolution and width of the reduced-volume interferogram, respectively. On the other hand, the interval and number of steps of the PZT determine the longitudinal resolution and width of the reduced-volume interferogram along the $Z$ axis, respectively. These transverse and longitudinal parameters of the reduced-volume interferogram are related to the $3 \mathrm{D}$ resolutions $\delta x, \delta y, \delta z$ and the spectral resolution $\Delta k / 2 \pi$.

Note that the spectral resolution $\Delta k / 2 \pi$ is determined by the reciprocal of the width of the volume interferogram along the $Z$ axis (i.e., $\Delta k / 2 \pi=l_{z}$ ). The transverse resolutions of the retrieved image along the $x$ - and $y$-directions are limited by the corresponding aperture sizes of the complex hologram, $l_{x}=l_{y}=l$, and the retrieval depth $z$. These resolutions, respectively, denoted $\delta x$ and $\delta y$, are expressed as $\delta x=\delta y=\lambda F$, where $F=z / l$ is the effective $f$-number of the experimental system and $\lambda=2 \pi / k$. In addition, the longitudinal resolution $\delta z$ is estimated as $\delta z=\lambda F^{2}$. This longitudinal resolution is proportional to the product of the transverse resolution, $\delta x$ or $\delta y$, and $F$. On the other hand, the fields of view along the $x$ - and $y$-directions, denoted $x_{\max }$ and $y_{\max }$, are expressed as $x_{\max }=z \lambda / \Delta l_{x}$ and $y_{\max }=z \lambda / \Delta l_{y}$, where $\Delta l_{x}$ and $\Delta l_{y}$ are the pixel sizes of the complex hologram in the $x$ and $y$ directions, respectively.

We also note that recovered holograms are taken only as positive $k$-components and complex ones. This suggests that the retrieved images are never contaminated by pseudoscopic images or by the 0th components. In this sense, the retrieved images are clean.

The specifications of the three planar light sources (S1, S2, and S3) are summarized in Table 1.

The measured object is composed of three planar light sources whose peak wavelengths are 643, 503 , and $450 \mathrm{~nm}$. The distance to these light sources measured from the observation plane are 50,61 , and $70 \mathrm{~mm}$, respectively.

\section{Experimental Result}

Figure 6 shows the reduced-volume (3D) interferogram obtained in our experiment. The intensity profile plotted along $Z$ axis at the center point of this reduced-volume interferogram is shown in Fig. 7 .

The continuous spectral profile over the observation plane that is acquired by taking the Fourier transform with respect to $Z$ along the center of the reduced-volume interferogram is shown in Fig. 8 . Three spectral peaks are located near $630,50 \overline{4}$, and $448 \mathrm{~nm}$. Within the spectral resolution, the retrieved spectral profile agrees with the separated spectral profile, which is shown in Fig. $\underline{4}$.

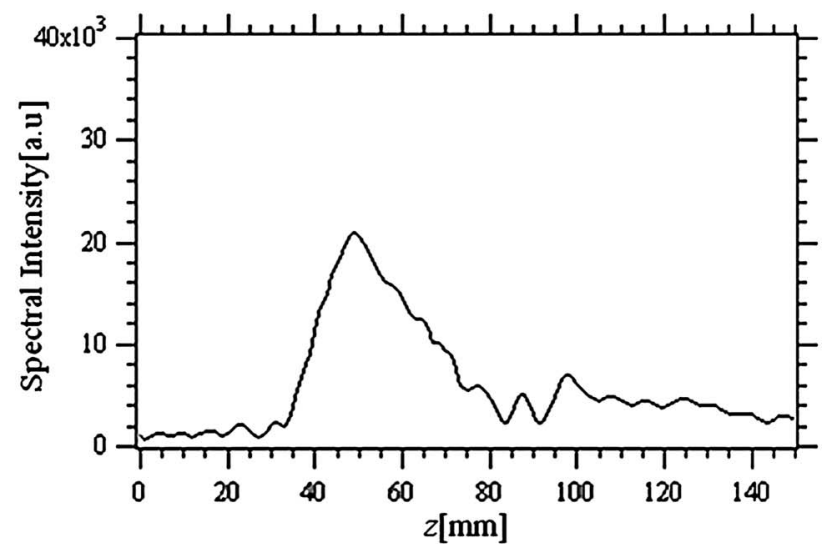

(a)

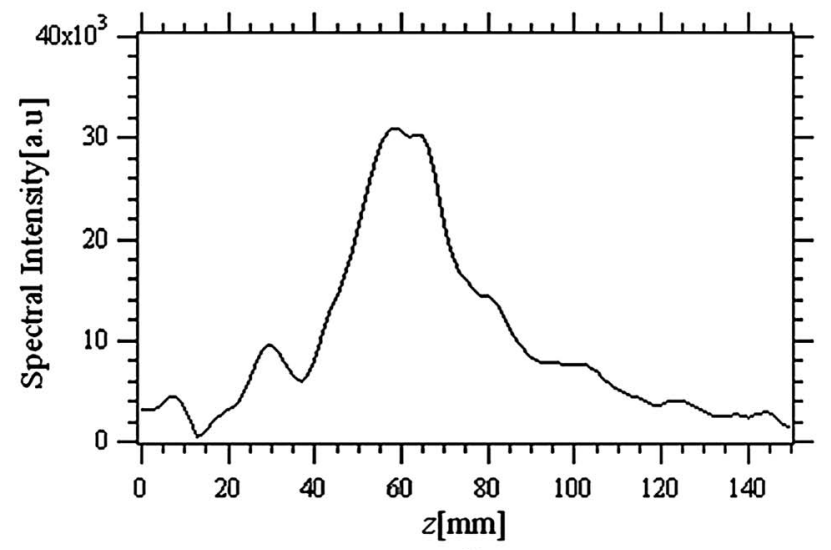

(b)

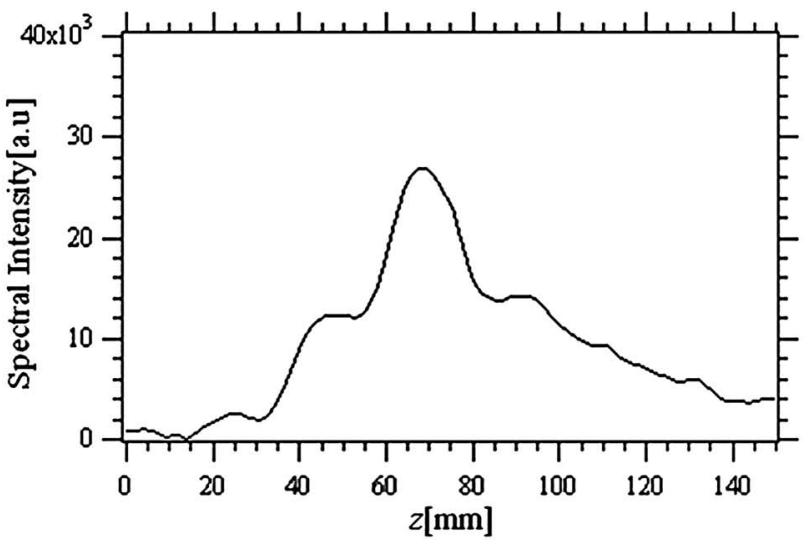

(c)

Fig. 12. Intensity profiles of the retrieved images along the $z$ axis: (a) S1, (b) S2, and (c) S3. 
Figures 9(a) and 9(b) show the phase distribution and absolute value of the cross-spectral density. That is essentially equivalent to the complex incoherent hologram for the corresponding spectral component of the object. Figure 9(c) shows the in-focus image for the light source $S \overline{1,}$ retrieved from the complex hologram at $50 \mathrm{~mm}$, measured from the observation plane. Similarly, those holograms and in-focus images for the light source S2 and S3 placed at 61 and $70 \mathrm{~mm}$ are shown in Figs. 10 and 11, respectively. From the intensity distributions of these images along the $x$ and $y$ axes, the sizes of the image in Fig. 9(c) are $0.53 \mathrm{~mm}$ (at $Y=-0.3$ ), $0.49 \mathrm{~mm}$ (at $X=0$ ); in Fig. $10(\mathrm{c})$ are $0.61 \mathrm{~mm}$ (at $Y=-0.05$ ), $0.41 \mathrm{~mm}$ (at $X=-0.05$ ); and in Fig. 11(c) are $0.49 \mathrm{~mm}$ (at $Y=0.0$ ), $0.51 \mathrm{~mm}$ (at $X=0.1$ ). Within the transverse resolution, we find that the results agree with the actual experimental condition. As a result, 3D images of three planar light sources with different shapes of cross sections are successfully retrieved by our method.

Figure 12 shows the intensity profile along the $z$ axis. The maximum intensity of three light sources (S1, S2, and S3) appear close to the actual distances 50,61 , and $70 \mathrm{~mm}$, respectively, from the observation plane. Thus, the depth information of the three planar light sources is successfully retrieved.

The measured spatially incoherent polychromatic object in the experiment was the three planar light sources with triangular and rectangular shapes, composed of three continuous spectra (see in Fig. 5). Figure 13 partly shows the retrieved spectral images for multiple spectral bands. We can clearly see that two triangular shapes having different continuous spectra and their positions are clearly visible at $\lambda=438.3-530.0 \mathrm{~nm}$. Similarly, the rectangular image at $\lambda=630 \mathrm{~nm}$ is clearly seen as well. These
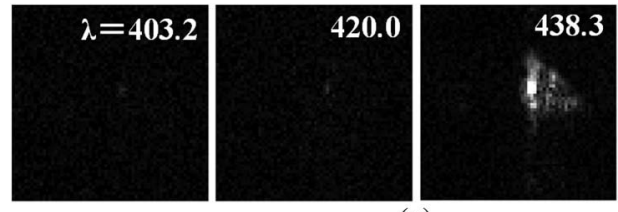

(a)
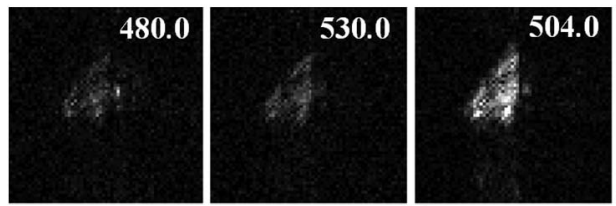

(b)
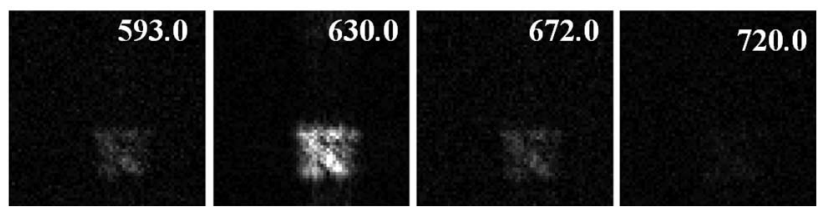

(c)

Fig. 13. Retrieved in-focus spectral images of the three planar light sources for multiple spectral bands. The object depths are as follows: (a) $70 \mathrm{~mm}$ for $\lambda=403.2-458.2 \mathrm{~nm}$, (b) $61 \mathrm{~mm}$ for $\lambda=480.0-560.0 \mathrm{~nm}$, and (c) $50 \mathrm{~mm}$ for $\lambda=593.0-720.0 \mathrm{~nm}$. results agree with the retrieved spectral density in Fig. 8.

Figure 14 shows the decomposed continuous spectra of the three planar light sources by tracing the variation of the images' intensities with respect to wavelength. In this manner, we may show the continuous spectrum at a particular point on the $3 \mathrm{D}$ images. This fact analyzes the applicability of our method to identify materials of each 3D point of the measured object by comparing the spectral

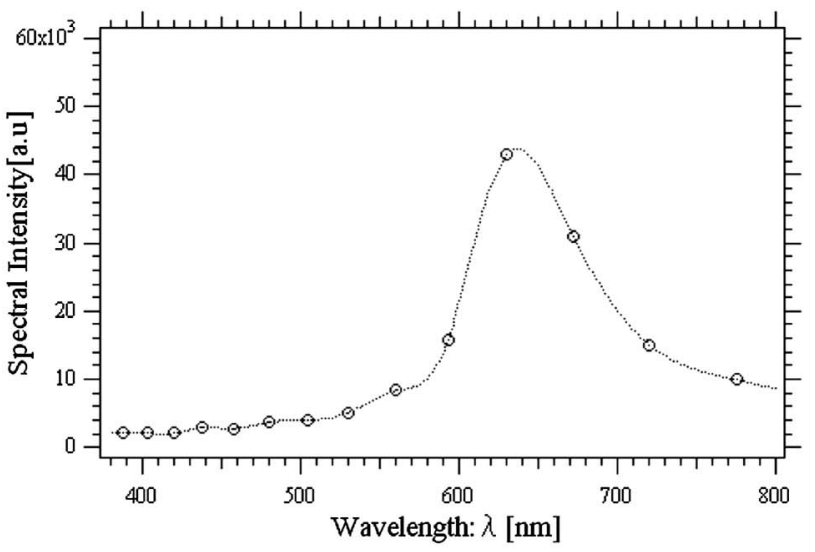

(a)

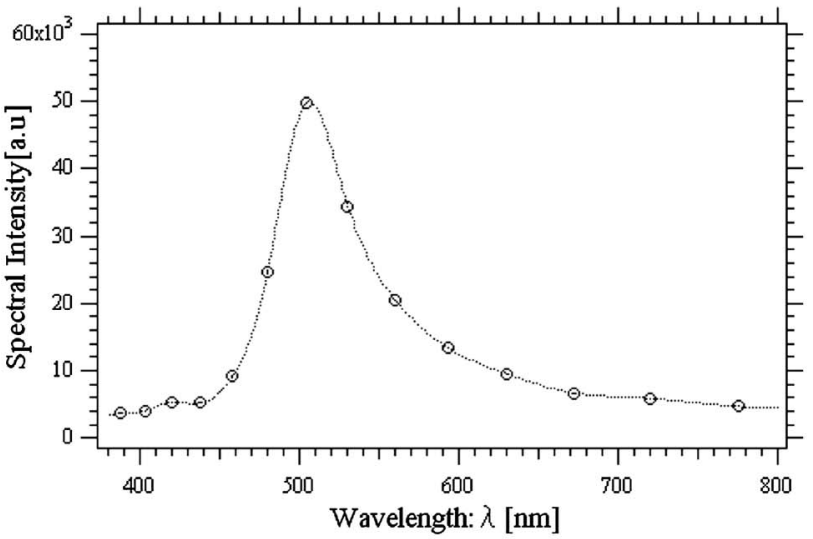

(b)

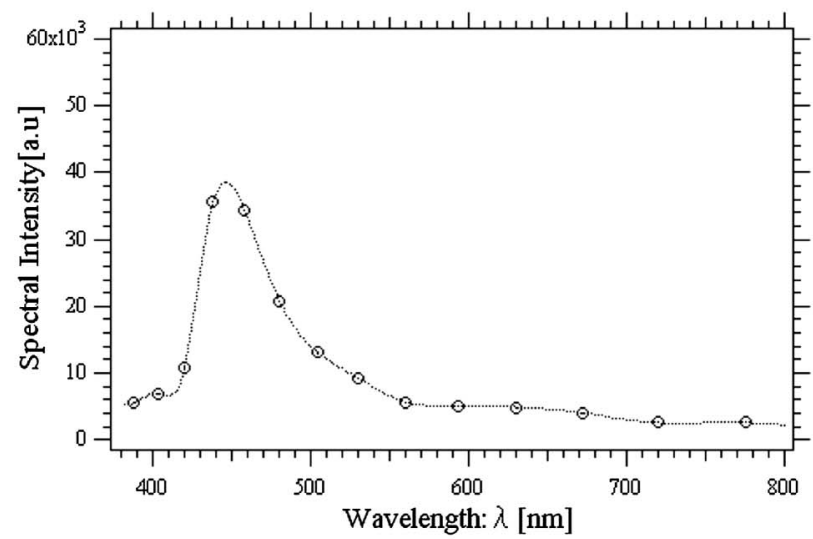

(c)

Fig. 14. Decomposed spectral profiles of the three planar light sources at the particular 3D spatial positions on the 3D images of those: (a) $z=50 \mathrm{~mm}$, (b) $z=61 \mathrm{~mm}$, and (c) $z=70 \mathrm{~mm}$. 
symmetries of the matter, at least in principle. Our experimental results agree well with the experimental setup. We may state that, by applying a two-wavefront folding interferometer and aperture synthesis, the $3 \mathrm{D}$ spatial information and spectral information for different shapes of three planar light sources with different continuous spectra, located at different positions (the polychromatic light source distribution), can be successfully acquired simultaneously.

\section{Conclusion}

We succeeded in retrieving a set of spectral components of 3D images for spatially incoherent, polychromatic light sources that were composed of three different planar light sources of different shapes having different continuous spectra. Consequently, we concluded that both 3D spatial information and spectral information of spatially incoherent light sources with different continuous spectra, located at different positions, can be obtained by our method, which uses a two-wavefront folding interferometer and signal processing, including aperture synthesis, spectral decomposition, and 3D image retrieval.

This work was supported in part by the Research Foundation for Opto-Science and Technology, and Grants-in-Aid for Scientific Research from the Japan Society for the Promotion of Science (22560031). In addition, the authors express their sincere appreciation to Dr. Akiko Hirai of the National Institute of Advanced Industrial Science and Technology for fruitful discussion and comments on this work.

\section{References}

1. J. Rosen and A. Yariv, "General theorem of spatial coherence: application to three-dimensional imaging," J. Opt. Soc. Am. A 13, 2091-2095 (1996).
2. D. L. Marks, R. A. Stack, and D. J. Brady, "Three-dimensional coherence imaging in the Fresnel domain," Appl. Opt. 38, 1332-1342 (1999).

3. H. Arimoto, K. Yoshimori, and K. Itoh, "Retrieval of the crossspectral density propagating in free space," J. Opt. Soc. Am. A 16, 2447-2452 (1999).

4. H. Arimoto, K. Yoshimori, and K. Itoh, "Interferometric threedimensional imaging based on retrieval of generalized radiance distribution," Opt. Rev. 7, 25-33 (2000).

5. K. Itoh and Y. Ohtsuka, "Fourier-transform spectral imaging: retrieval of source information from three-dimensional spatial coherence," J. Opt. Soc. Am. A 3, 94-100 (1986).

6. K. Itoh, T. Inoue, T. Yoshida, and T. Ichioka, "Interferometric supermultispectral imaging," Appl. Opt. 29, 1625-1630 (1990).

7. K. Yoshimori and K. Itoh, "Interferometry and radiometry," J. Opt. Soc. Am. A 14, 3379-3387 (1997).

8. D. L. Marks, M. Fetterman, R. A. Stack, and D. J. Brady, "Spectral tomography from spatial coherence measurements," Proc. SPIE 3020, 48-55 (2000).

9. K. Yoshimori, "Interferometric spectral imaging for threedimensional objects illuminated by a natural light source," J. Opt. Soc. Am A 18, 765-770 (2001).

10. K. Yoshimori, "Passive digital multispectral holography based on synthesis of coherence function," Proc. SPIE 6252, 625221 (2006).

11. K. Yoshimori and M. Sasamoto, "Experimental report on fully interferometric three-dimensional imaging spectroscopy," in Digital Holography and Three-Dimensional Imaging, OSA Technical Digest (CD) (Optical Society of America, 2009), paper DWB34.

12. S. Teeranutranont and K. Yoshimori, "Application of digital holographic three-dimensional imaging spectrometry to a spatially incoherent, polychromatic object," in Digital Holography and Three-Dimensional Imaging, OSA Technical Digest (CD) (Optical Society of America, 2011), paper DWB36.

13. K. Yoshimori, "Digital holographic three-dimensional imaging spectrometry," in Digital Holography and Three-Dimensional Imaging, OSA Technical Digest (CD) (Optical Society of America, 2012), paper DW1C.1.

14. M. Sasamoto and K. Yoshimori, "First experimental report on fully passive interferometric three-dimensional imaging spectrometry," Jpn. J. Appl. Phys. 48, 09LB03-1-4 (2009).

15. M. Sasamoto and K. Yoshimori, "Three-dimensional imaging spectrometry by fully passive interferometry," Opt. Rev. 19, 29-33 (2012). 